\title{
Strain-Induced Charge Transfer and Polarity Control of a Heterosheet Comprising $\mathrm{C}_{60}$ and Graphene
}

\author{
Yamato A. Saucier, Susumu Okada, and Mina Maruyama* \\ Graduate School of Pure and Applied Sciences, University of Tsukuba, 1-1-1 Tennodai, Tsukuba
}

305-8571, Japan

\begin{abstract}
Using density functional theory combined with the effective screening medium method, the energetics and electronic structure of $\mathrm{C}_{60}$ molecular sheet adsorbed on graphene were studied in terms of biaxial strains. The optimum spacing and interlayer interaction monotonically decreases and increases, respectively, with increasing biaxial tensile strain. The biaxial compressive strain induces electron transfer from the graphene to the $\mathrm{C}_{60}$ at a $2 \%$ lateral compression, leading to an all-carbon charge transfer complex. The heterosheet possesses an intrinsic dipole moment along the graphene-to- $\mathrm{C}_{60}$ molecular layer direction.
\end{abstract}

Nanoscale carbon materials such as fullerenes, ${ }^{1-3)}$ carbon nanotubes $^{4-6)}$ and graphene ${ }^{7,8)}$ have been attracting much attention in the three decades since the first prediction of the existence of hollowcage structure of $\mathrm{C}_{60}$ from the mass spectra of carbon clusters. These carbon allotropes are characterized by the dimensionality of their covalent networks associated with the $\sigma$ electrons of three-fold coordinated (approximately $\mathrm{sp}^{2}$ ) $\mathrm{C}$ atoms, which allow these allotropes to be stable constituent materials for future nanotechnologies. ${ }^{9}{ }^{10)}$ Chemically functionalized fullerenes and carbon nanotubes can respectively act as an acceptor layer for organic thin-film photovoltaic devices ${ }^{11-13)}$ and as conducting channels for flexible field effect transistors. ${ }^{14-16)}$ The versatile application possibilities of these nanocarbon materials are ascribed to the variations in their electronic properties, which depend on their local network topology and the boundary conditions imposed on the $\mathrm{sp}^{2}$ covalent networks. For example, carbon nanotubes are either a metal or semiconductor depending on their diameter and chirality. ${ }^{5,6)}$ Zigzag edge of graphene causes spin polarization owing to the delicate balance of electron transfer near the edge atomic sites. ${ }^{17-19)}$

The planar, tubular or hollow-cage covalent network of a nanocarbon material causes it to be chemically inert and stable under ambient conditions. Therefore, these nanocarbon materials have also been attracting much attention as building blocks for hierarchical solids, an example of which is fullerenes and nanotubes forming solid phases with close-packed structures. ${ }^{20-25)}$ In such hierarchical solids, the nanoscale spacing arising from these nanoscale covalent networks allows them host materials for the intercalation of atoms and molecules, which results in the generation of derivatives that

*E-mail: mmaruyama@comas.frsc.tsukuba.ac.jp 
exhibit unusual physical phenomena. In addition to homogeneous hierarchical structures, heterogeneous hybrid structures with mixed dimensionality have been also proposed and synthesized. For instance, an intercalation compound consisting of $\mathrm{C}_{60}$ and graphite has been theoretically predicted and experimentally synthesized. ${ }^{26,27)}$ Also, fullerenes have been encapsulated inside carbon nanotubes, leading to nanoscale peapods that are characterized by a hybrid of their zero- and one-dimensional covalent networks bound via weak inter-unit interactions. ${ }^{28-30)}$ Experimental and theoretical works have demonstrated that these complexes comprising mixed dimensionality in their covalent networks exhibit unusual physical properties not present in each constituent, which are owing to the difference in the local atomic structure of the constituents and the nanoscale spacing between them. ${ }^{20,24,25,31,32 \text { ) }}$

Recent advancements in synthetics and the fabrication techniques of nanocarbon materials and their hybrids further extends the science and technology associated with these nanoscale complexes. The exfoliation and transcription of graphene and other layered materials allow us to handle the heterostructures of layered materials. ${ }^{33-35)}$ In such complexes, interplay betwixt the confined nanospacing between the constituents and the vacuum outside the dense covalent networks may bestow further variations in the physical properties of the complex. In the present work, we focus on a heterosheet possessing an ultimate thickness, comprising graphene and a $\mathrm{C}_{60}$ molecular layer as the thinnest version of the $\mathrm{C}_{60}$-graphite intercalation compound. With this heterosheet, we investigate the unusual physical properties that arise from the hybrid structure using density functional theory (DFT) combined with the effective screening medium (ESM) method. Biaxial compressive strain is found to induce electron transfer from graphene to the lowest unoccupied state of $\mathrm{C}_{60}$, leading to an all-carbon charge transfer complex. Furthermore, owing to the difference in the work function of graphene and $\mathrm{C}_{60}$, the sheet exhibits a polarity normal to the layers whose value is tunable by biaxial compressive and tensile strains. Calculations also demonstrate that the van der Waals (vdW) interaction and the optimum spacing between the $\mathrm{C}_{60}$ layer and graphene are approximately proportional to the biaxial strain.

All calculations were performed using DFT ${ }^{36,37)}$ implemented in the STATE package. ${ }^{38)}$ For the calculation of the exchange-correlation energy amongst the interacting electrons, the generalized gradient approximation including the spin degree of freedom was used with the functional forms of Perdew-Burke-Ernzerhof. ${ }^{39)}$ To describe the weak dispersive interaction between $\mathrm{C}_{60}$ and graphene, we considered the vdW interaction by treating vdW-DF2 with the C09 exchange-correlation functional. ${ }^{40-42)}$ An ultrasoft pseudopotential generated with the Vanderbilt scheme was used to describe electron-ion interactions. ${ }^{43}$ The valence wave functions and deficit charge density were expanded using plane-wave basis sets with cutoff energies of 25 and $225 \mathrm{Ry}$, respectively. Structural optimization was performed until the remaining forces on each atom were less than $5 \mathrm{mRy} / \AA$ under each lateral lattice constant. Brillouin-zone integration was performed with the $\Gamma$-centered $4 \times 4 \times 1$ uniform $k$-mesh for self-consistent electronic structure calculations of the heterosheet comprising a $\mathrm{C}_{60}$ molecular layer and graphene. To simulate the heterosheet, $\mathrm{C}_{60}$ formed a triangular lattice whose cell parameter corre- 

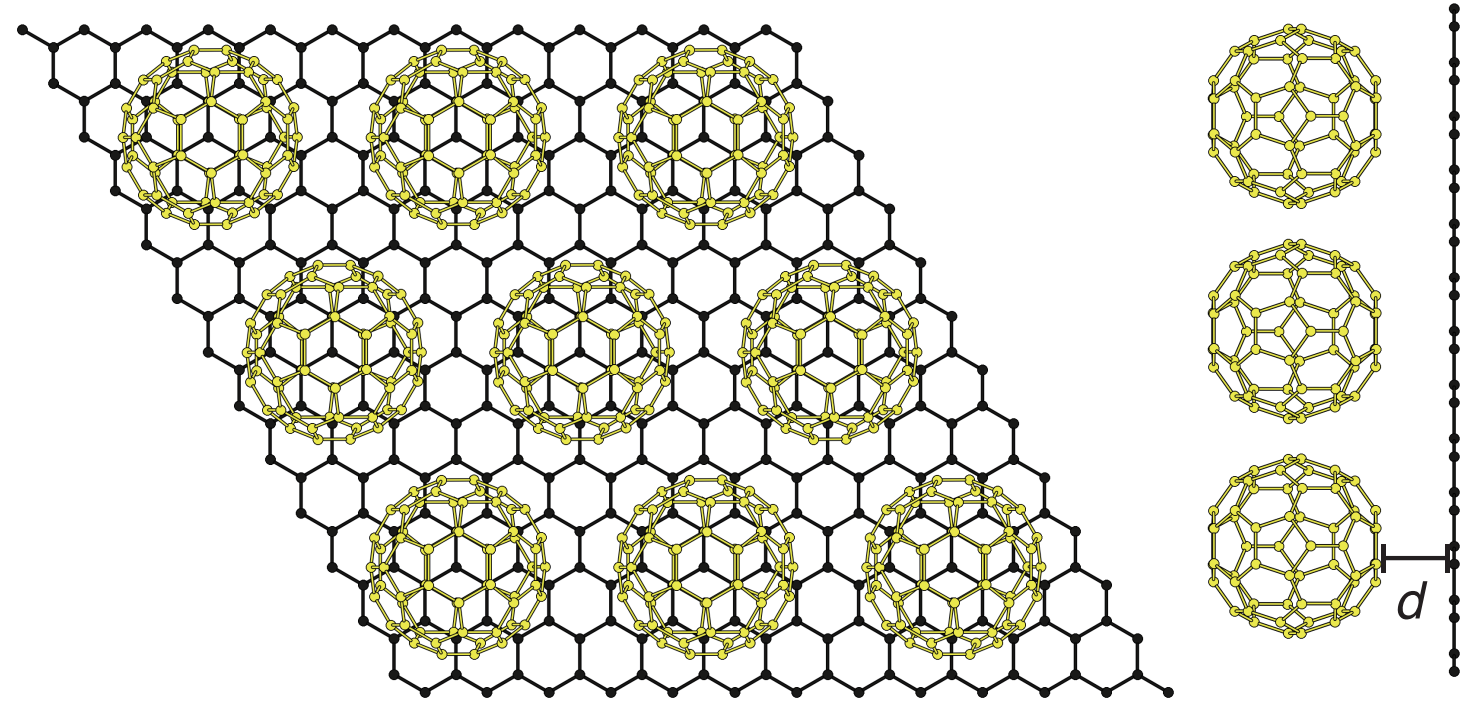

Fig. 1. (Color online) Top and side views of an optimized heterosheet structure consisting of a $\mathrm{C}_{60}$ molecular layer and graphene, where $d$ indicates the optimum spacing between $\mathrm{C}_{60}$ and graphene. The $\mathrm{C}$ atoms belonging to graphene (black spheres) and $\mathrm{C}_{60}$ (yellow spheres) are indicated.

sponded to the $4 \times 4$ lateral periodicity of graphene (Fig. 1). Under biaxial strain, the cell parameters for both graphene and $\mathrm{C}_{60}$ were simultaneously scaled to retain the commensurability condition between the $\mathrm{C}_{60}$ sheet and graphene. Because an intrinsic dipole moment normal to the heterosheet is expected to occur, we adopted the ESM method to avoid an unphysical dipole interaction with the imagining cells normal to the sheet, which is separated by a vacuum spacing of at least $10 \AA$ vacuum spacing, under periodic boundary conditions. ${ }^{44)}$

Figure 2 shows the optimized spacing and binding energy between the $\mathrm{C}_{60}$ molecular layer and graphene as a function of the lateral lattice constant. The optimum spacing between the $\mathrm{C}_{60}$ layer and graphene monotonically increases with increasing the lattice parameter. Simultaneously, the binding energy also depends on the lateral lattice constant, where the interlayer binding energy increases as the biaxial compressive strain decreases. Thus, the biaxial strain can control the interlayer spacing and binding energy of a heterosheet consisting of a $\mathrm{C}_{60}$ molecular layer and graphene. The decrease of the interlayer binding energy under the tensile strain is ascribed to a decrease of the charge density of the graphene network owing to bond elongation. The charge densities of the covalent bond of graphene under a $10 \%$ tensile and compressive strain are lower by $0.05 e /$ a.u. and higher by 0.10 $e /$ a.u., respectively, than that under the equilibrium lateral lattice constant.

Because $\mathrm{C}_{60}$ possesses a large electron affinity owing to its deep lowest unoccupied state arising from the pentagonal rings, the heterosheet is expected to possess an unusual electronic structure. Figure 3 shows the electronic structures of a heterosheet that is under equilibrium, and one that has compressed and elongated lateral lattice parameters. With the equilibrium lattice parameter, the lowest 
(a)

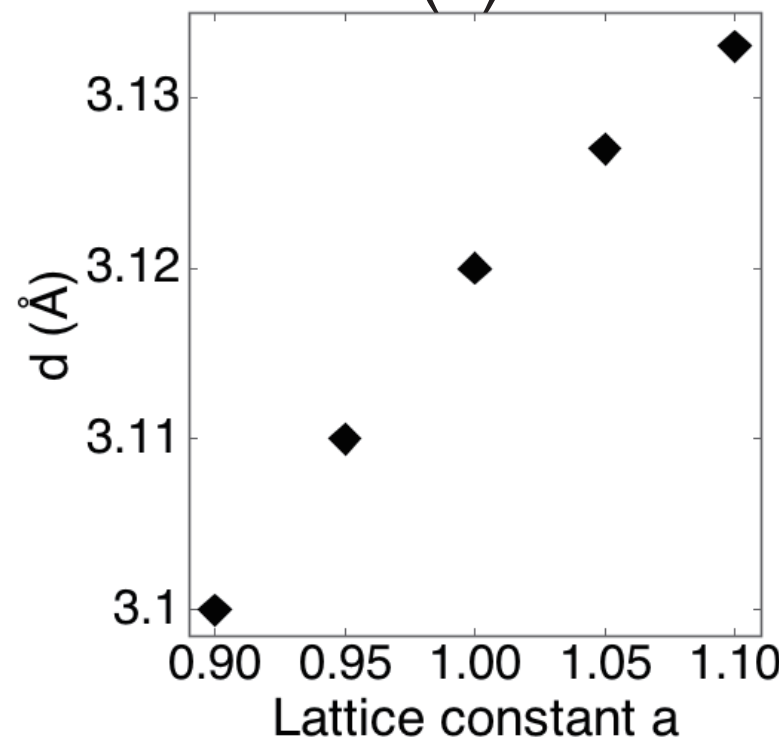

(b)

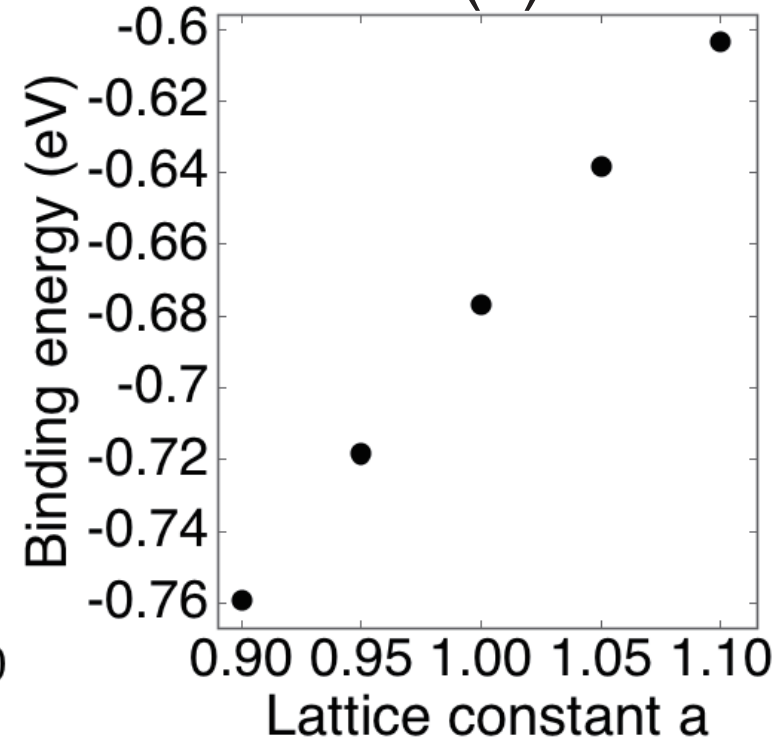

Fig. 2. (Color online) (a) Optimized spacing and (b) binding energy between $\mathrm{C}_{60}$ and graphene as a function of the lateral lattice constant $a\left(1 / a_{0}\right)$, where $a_{0}$ corresponds to the experimental lateral lattice constant of graphene with a $4 \times 4$ lateral cell $\left(a_{0}=9.838 \AA\right)$.

(a)

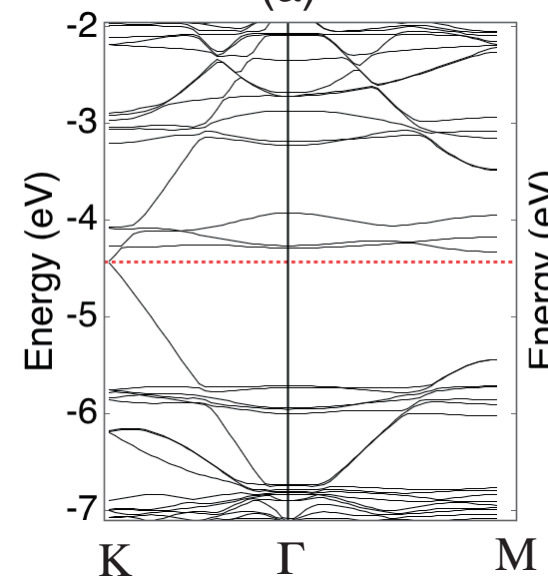

(b)

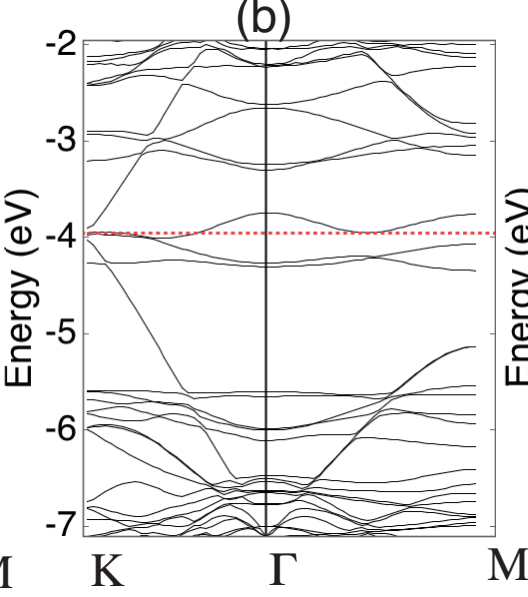

(c)

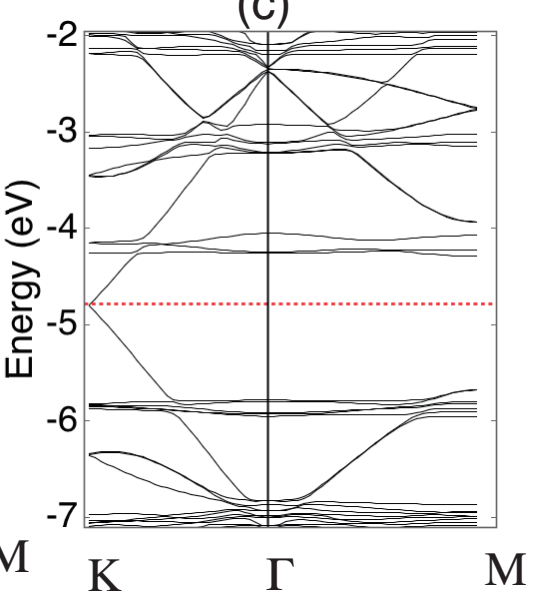

Fig. 3. Electronic structures of the heterosheet consisting of a $\mathrm{C}_{60}$ molecular layer and graphene under the lateral lattice constant of (a) $a_{0}=9.838 \AA$, (b) $a=0.95 a_{0}$, and (c) $a=1.05 a_{0}$. The horizontal dotted lines denote the Fermi level.

unoccupied states ( $t_{1 u}$ state) are located just above the Dirac cone or the Fermi level. This agrees with the case of the bulk $\mathrm{C}_{60}$ graphite intercalation compound but deviates from that of the $\mathrm{C}_{60}$ peapods in which the $t_{1 u}$ state crosses the Fermi level. Under a biaxial compressive strain, the $t_{1 u}$ state crosses the Fermi level and the Dirac cone, thereby simultaneously increasing their bandwidth owing to the 
(a)

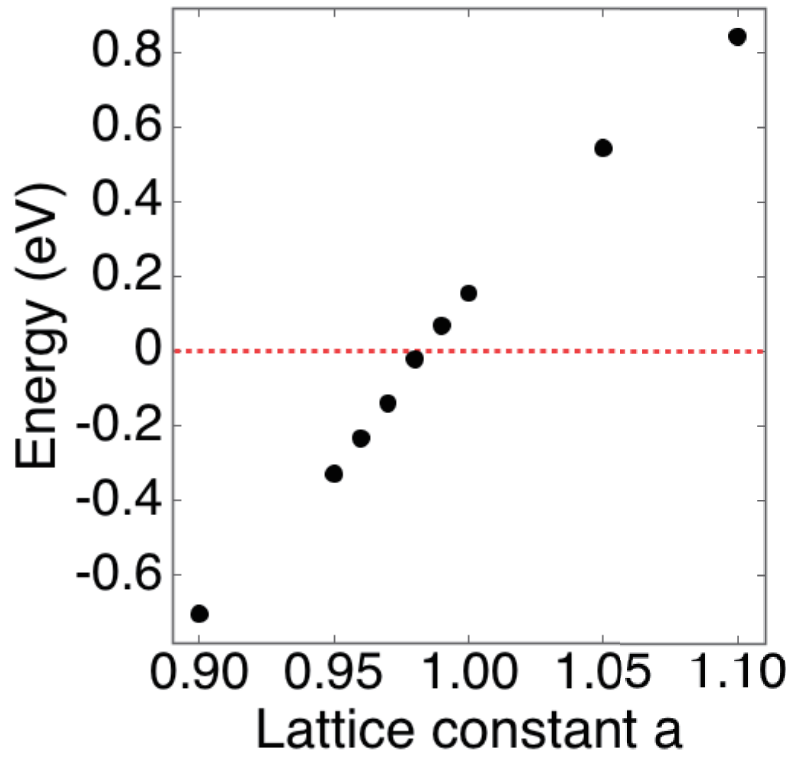

(b)

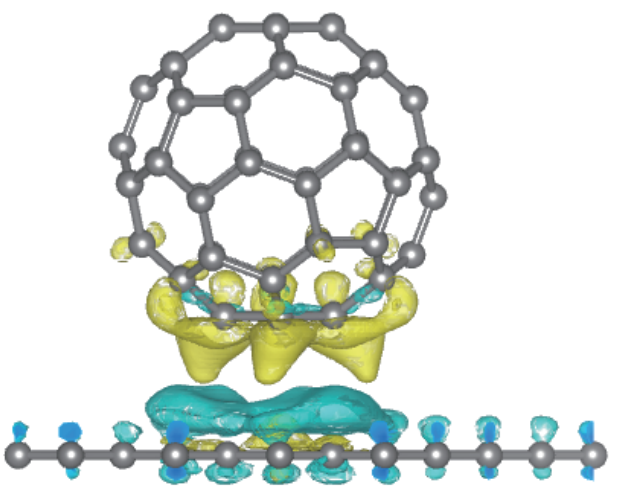

Fig. 4. (a) Eigenvalue of the $t_{1 u}$ state with respect to that of the Dirac cone as a function of the lateral lattice constant. (b) Isosurfaces of the accumulated and depressed charge densities formed on the heterosheet under compressive strain with a lattice constant of $a=0.95 a_{0}$, where $a_{0}$ is the experimental lattice constant of graphene with $4 \times 4$ lateral periodicity. The isosurfaces are denoted as negatively- (yellow) or positively- (blue) charged.

decrease of the inter- $\mathrm{C}_{60}$ spacing. The characteristic electronic structure indicates that the electron is transferred from graphene to the $t_{1 u}$ state of $\mathrm{C}_{60}$, leading to a charge transfer complex that consists only of $\mathrm{C}$ atoms. The band structure modulation is ascribed to an upward shift of the Dirac cone of graphene, which is owing to the increase of the charge density of graphene network that occurs under compressive strain. In contrast, the Dirac cone shifts downward with a biaxial tensile strain, leading to a higher $t_{1 u}$ state with respect to the Dirac cone.

To determine the critical compressive strain at which electron transfer occurs, the $t_{1 u}$ energy with respect to the Dirac point energy [Fig. 4(a)] was investigated, and it was found that the $t_{1 u}$ energy monotonically decreases (increases) as the lattice constant decreases (increases) from the equilibrium lattice constant value. Further, the $t_{1 u}$ state crosses the Dirac point energy at the critical compressive strain of $2 \%$ and, finally, the $t_{1 u}$ is located $0.7 \mathrm{eV}$ below the Dirac point at the compressive strain of $10 \%$. Fig. 4(b) shows the isosurfaces of electron accumulation and depression, $\Delta \rho(\boldsymbol{r})$, of the heterosheet under 5\% compressive strain. The accumulation and depression of the charge density $\Delta \rho(\boldsymbol{r})$ is evaluated by the charge density difference between the complex and the sum of an isolated $\mathrm{C}_{60}$ sheet and a graphene layer. The distribution of $\Delta \rho(\boldsymbol{r})$ corroborates the electron transfer from graphene to $\mathrm{C}_{60}$ in the heterosheet structure on the grounds that the electron density at the lower part of the $\mathrm{C}_{60}$ increases while that on the graphene decreases for the heterosheet structure compared to their isolated form. The injected electron in $\mathrm{C}_{60}$ may cause the superconductivity as the case of alkali metal 
(a)

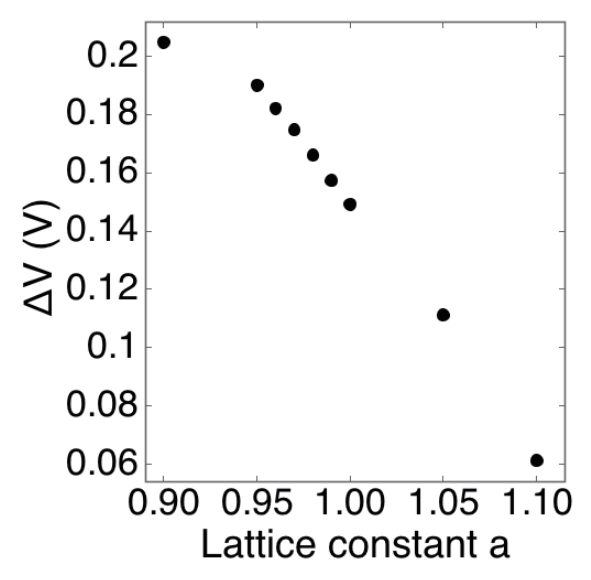

(b)

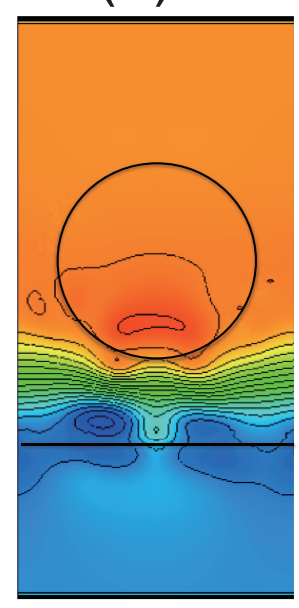

(c)

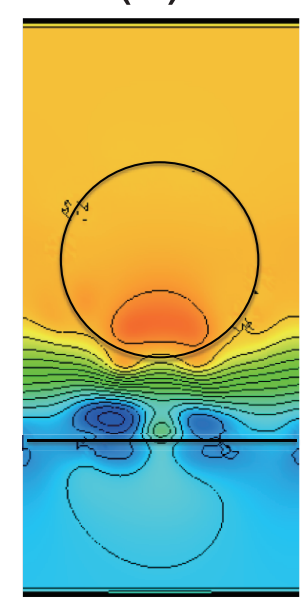

(d)

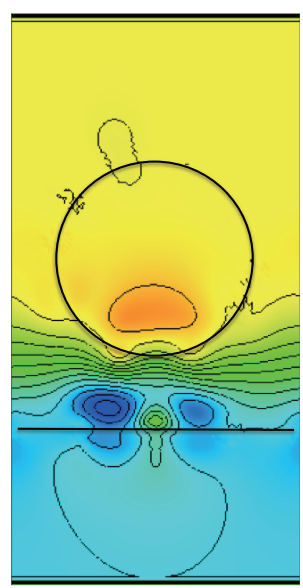

Fig. 5. (a) The potential difference between the vacuums of the $\mathrm{C}_{60}$ and graphene surface as a function of the lateral lattice constant. Contour plots of the electrostatic potential of the heterosheet consisting of $\mathrm{C}_{60}$ and graphene with the lateral lattice parameters of (b) $a=0.95 a_{0}$, (c) $a=a_{0}$ and (d) $a=1.05 a_{0}$. Red, yellow, and blue colors correspond the high, moderate, and low electrostatic potential regions, respectively. The solid circles and lines indicate the atomic positions of $\mathrm{C}_{60}$ and graphene, respectively.

doped $\mathrm{C}_{60}$ fullerides, even though the system sorely consist of carbon atoms. On the other hand, the narrow band width of $t_{1 u}$ states may also cause spin polarization on $\mathrm{C}_{60}$, allowing the heterosheets a constituent material for spintronic devices of which functionality is tuned by the mechanical strain.

The bond alternation arising from the pentagonal rings in the fullerene cages causes dipole or multipole moments on the cages, ${ }^{45)}$ and therefore the heterosheet comprising $\mathrm{C}_{60}$ and graphene also exhibits polar properties normal to the constituent layers. Figure 5(a) shows the electrostatic potential difference between two cell boundaries normal to the heterosheet as a function of the lattice constant. The sheet possesses a finite electrostatic potential difference between $\mathrm{C}_{60}$ and graphene, wherein the potential energy at the $\mathrm{C}_{60}$ layer is higher by $0.145 \mathrm{~V}$ than that at graphene and thus leads to an electric field of approximately $40 \mathrm{mV} / \AA$ 品 between the two layers. The polarity increase (decrease) with decreasing (increasing) lattice parameters indicates that a biaxial strain on the heterosheet can tune the electricity induced in the heterosheet. The fact indicate that the heterosheet could be applicable for the variable voltage electric power supply of which voltage can be tuned by the external mechanical strains. In addition to the electrostatic potential difference along the direction normal to the layers, the electron accumulation and depression around the interface region modulates the electrostatic potential parallel to the layer [Figs. 5(b)-(d)]. In other words, the electric field is concentrated around the interfaces between $\mathrm{C}_{60}$ and graphene, even though charge transfer is still absent [Figs. 5(c) and (d)].

In summary, using DFT combined with the ESM method we investigated the electronic and geometric structures of a heterosheet consisting of a $\mathrm{C}_{60}$ molecular layer and graphene, which represents the thinnest version of the $\mathrm{C}_{60}$-graphite intercalation compound. Our calculations showed that electron 
transfer is induced by the biaxial compressive strain of $2 \%$ lattice compression owing to the upward shift of the Dirac point of graphene arising from the increase of the electron density in the graphene layer. Further, the electron transfer monotonically increases with the compressive strain. The charge density modulation in the covalent bond of graphene induced by the strains also leads to a modulation of the optimum spacing and interlayer binding energy between the $\mathrm{C}_{60}$ layer and graphene. Also, a work function difference between the graphene and $\mathrm{C}_{60}$ sheets induces an electrostatic potential difference between these layers, leading to a polarity normal to the heterosheet that is also tunable by the biaxial strain.

Acknowledgment This work was supported by JST-CREST Grant Number JPMJCR1532 from the Japan Science and Technology Agency, JSPS KAKENHI Grant Numbers JP17H01069, JP16H00898, and JP16H06331 from Japan Society for the Promotion of Science, and the Joint Research Program on Emission Energy Research, Institute of Advanced Energy, Kyoto University. A part of the calculations was performed on an NEC SX-Ace at the Cybermedia Center at Osaka University and on an SGI ICE XA/UV at the Institute of Solid State Physics, The University of Tokyo. 


\section{References}

1) H. W. Kroto, J. R. Heath, S. C. O’Brien, R. F. Curl, and R. E. Smalley, Nature 318, 162 (1985).

2) W. Krätschmer, K. Fostiropoulous, and D. R. Hoffman, Chem. Phys. Lett. 170, 167 (1990).

3) W. Krätschmer, L. D. Lamb, K. Fostiropoulous, and D. R. Hoffman, Nature 347, 354 (1990).

4) S. Iijima, Nature 354, 56 (1991).

5) N. Hamada, S. Sawada, and A. Oshiyama, Phys. Rev. Lett. 68, 1579 (1992).

6) R. Saito, M. Fujita, M. S. Dresselhaus, and G. Dresselhaus, Appl. Phys. Lett. 60, 2204 (1992).

7) K. S. Novoselov, A. K. Geim, S. V. Morozov, D. Jiang, M. I. Katsnelson, I. V. Grigorieva, S. V. Dubonos, and A. A. Firsov, Nature 438, 197 (2005).

8) C. Berger, Z. Song, X. Li, X. Wu, N. Brown, C. Naud, D. Mayou, T. Li, J. Hass, A. N. Marchenkov, E. H. Conrad, P. N. First, and W. A. de Heer, Science 312, 1191 (2006).

9) M. S. Dresselhaus, G. Dresselhaus, and P. C. Eklund, Science of Fullerenes and Carbon Nanotubes (Academic Press, San Diego, CA, 1996).

10) A. H. Castro Neto, F. Guinea, N. M. R. Peres, K. S. Novoselov, and A. K. Geim, Rev. Mod. Phys. 81, 109 (2009).

11) Y. Matsuo and E. Nakamura, Chem. Rev. 108, 3016 (2008).

12) Y. Matsuo, Y. Sato, T. Niinomi, I. Soga, H. Tanaka, and H. Nakamura, J. Am. Chem. Soc. 131 (44), 16048 (2009).

13) Y. Matsuo, Chem. Lett. 41, 754 (2012).

14) S. J. Tans, A. R. M. Verschueren, and C. Dekker, Nature 393, 49 (1998).

15) R. Martel, T. Schmidt, H. R. Shea, T. Hartel, and Ph. Avouris, Appl. Phys. Lett. 73, 2447 (1998).

16) Y. Nosho, Y. Ohno, S. Kishimoto, and T. Mizutani, Appl. Phys. Lett. 86, 073105 (2005).

17) M. Fujita, K. Wakabayashi, K. Nakada, and K. Kusakabe, J. Phys. Soc. Jpn. 65, 1920 (1996).

18) K. Nakada, M. Fujita, G. Dresselhaus, and M. S. Dresselhaus, Phys. Rev. B 54, 17954 (1996).

19) S. Okada and A. Oshiyama, Phys. Rev. Lett. 87, 146803 (2001).

20) S. Saito and A. Oshiyama, Phys. Rev. Lett. 66, 2637 (1991).

21) R. M. Fleming, T. Siegrist, P. M. Marsh, B. Hessen, A. R. Kortan, D. W. Murphy, R. C. Haddon, R. Tycko, G. Dabbagh, A. M. Mujsce, M. L. Kaplan, and S. M. Zahurak, Proc. Mat. Res. Soc. 206, 691 (1991).

22) K. Prassides, H. W. Kroto, R. Taylor, D. R. M. Walton, W. I. F. David, J. Tomkinson, R. C. Haddon, M. J. Rosseinsky, and D. W. Murphy, Carbon 30, 1277 (1992).

23) A. Thess, R. Lee, P. Nikolaev, H. Dai, P. Petit, J. Robert, C. Xu, Y. H. Lee, S. G. Kim, A. G. Rinzler, D. T. Colbert, G. E. Scuseria, D. Tománek, J. E. Fischer, and R. E. Smalley, Science 273, 483 (1996). 
24) J.-C. Charlier, X. Gonze, and J.-P. Michenaud, Europhys. Lett. 29, 43 (1995).

25) P. Delaney, H. J. Choi, J. Ihm, S. G. Louie, and M. L. Cohen, Nature 391, 466 (1998).

26) S. Saito and A. Oshiyama, Phys. Rev. B 49, 18413 (1994).

27) E. V. Rut'kov, A. Ya. Tontegode, and M. M. Usufov, Phys. Rev. Lett. 74, 758 (1995).

28) B. W. Smith, M. Monthioux, and D. E. Luzzi, Nature 396, 323 (1998).

29) B. Burteaux, A. Claye, B. W. Smith, M. Monthioux, D. E. Luzzi, and J. E. Fischer, Chem. Phys. Lett. 310, 21 (1999).

30) B. W. Smith, M. Monthioux, and D. E. Luzzi, Chem. Phys. Lett. 315, 31 (1999).

31) S. Okada, S. Saito, and A. Oshiyama, Phys. Rev. Lett. 86, 3835 (2001).

32) M. Otani, S. Okada, and A. Oshiyama, Phys. Rev. B 68, 125424 (2003).

33) A. K. Geim and I. V. Grigorieva, Nature 499, 419 (2013).

34) D. Jariwala, T. J. Marks, and M. C. Hersam, Nature Mater. 16, 170 (2017).

35) L. A. Chernozatonskii, A. G. Kvashnin, and P. B. Sorokin, Nanotechnology 27, 36520 (2016).

36) P. Hohenberg and W. Kohn, Phys. Rev. 136, B864 (1964).

37) W. Kohn and L. J. Sham, Phys. Rev. 140, A1133 (1965).

38) Y. Morikawa, K. Iwata, and K. Terakura, Appl. Surf. Sci. 169-170, 11 (2001).

39) J. P. Perdew, K. Burke, and M. Ernzerhof, Phys. Rev. Lett. 77, 3865 (1997).

40) K. Lee, É. D. Murray, L. Kong, B. I. Lundqvist, and D. C. Langreth, Phys. Rev. B 82, 081101(R) (2010).

41) V. R. Cooper, Phys. Rev. B 81, 161104(R) (2010).

42) I. Hamada and M. Otani, Phys. Rev. B 82, 153412 (2010).

43) D. Vanderbilt, Phys. Rev. B 41, 7892 (1990).

44) M. Otani and O. Sugino, Phys. Rev. B 73, 115407 (2006).

45) J. Sorimachi and S. Okada, Chem. Phys. Lett. 659, 1 (2016). 